\title{
Multiple genus 2 Heegaard splittings: a missed case
}

\author{
JOHN BERGE \\ MARTIN SCHARLEMANN
}

\begin{abstract}
A gap in a paper by Rubinstein and the second author [5] is explored: new examples are found of closed orientable 3-manifolds with possibly multiple genus 2 Heegaard splittings. Properties common to all the examples in that paper are not universally shared by the new examples: some of the new examples have Hempel distance 3, and it is not clear that a single stabilization always makes the multiple splittings isotopic.
\end{abstract}

$57 \mathrm{M} 15,57 \mathrm{~N} 10$

\section{Introduction}

In 1998, Rubinstein and the second author [5] studied the question of when there could be more than one distinct genus 2 Heegaard splitting of the same closed orientable 3-manifold. The goal of the project was modest: to provide a complete list of ways in which such multiple splittings could be constructed, but with no claim that each example on the list did in fact have multiple non-isotopic splittings (there could be isotopies from one splitting to another that are not apparent). Nor was there a claim that the list had no redundancies; a 3-manifold and its multiple splittings might appear more than once on the list. Such a list would still be useful, for if every example on the list could be shown to have a certain property, then that property would be true for any closed orientable 3-manifold $M$ that has multiple genus 2 splittings. Two examples were given in [5]:

- If $M$ is atoroidal then the hyperelliptic involutions determined by the two genus 2 Heegaard splittings commute.

- Any two genus 2 Heegaard splittings of $M$ become isotopic after a single stabilization.

Despite this modest goal, the argument in [5] contains a gap ${ }^{1}$. In 2008, the first author discovered a class of examples that do not appear on the list and which, moreover, have

\footnotetext{
${ }^{1}$ The error is on page 533: The last sentence of the first paragraph of Case 2 should have read, "The same curves cannot then be twisted in $X$ since $M$ is hyperbolike." This leaves open an additional possibility for $P_{X}, P_{Y}$, see the paper by the second author [7].
} 
mathematical properties that distinguish them in important ways from the examples that do appear in [5]. It is true that, even for the new examples, the hyperelliptic involutions commute. But we know of no argument showing that the new examples all share the second property above; that is, we cannot show that the newly discovered multiple splittings necessarily become isotopic after a single stabilization (though they do after two stabilizations).

A third property, shared by all examples in [5] but not by some of the new examples, is not listed above because the notion of Hempel distance of Heegaard splittings (see Hempel [3]) did not exist at the time [5] was written. But a retrospective look (see Section 6 below) will verify that all the splittings described in [5] have Hempel distance no greater than 2, whereas results of the first author [2] illustrate that at least some of the new examples have Hempel distance 3. (This also verifies that the gap in the argument in [5] actually led to missed examples.)

Here is an outline: In Section 2 we describe a general method for constructing closed orientable 3-manifolds that appear to have multiple genus 2 Heegaard splittings; these examples (called Dehn-derived) are based around Dehn surgery on a pair of strategically placed curves. It is shown in [7] that these examples do fill the gap in [5]. It follows from the construction that the hyperelliptic involutions of the alternate splittings always coincide.

It is not immediately obvious that curves supporting Dehn-derived examples can be found, but in Sections 3 and 4 we give three specific classes of examples. The classes are denoted $M_{H}$ (Section 3), $M_{\times I}$ and $M_{\text {hybrid }}$ (Section 4). ( $M_{H}$ can be viewed as a third variation of [5, Example 4.2].) For the examples $M_{H}$ and $M_{\text {hybrid }}$ a single stabilization suffices to make the alternate splittings equivalent, but this property is at least not apparent in most cases of $M_{\times I}$.

In Section 5 it is shown, using new results of the first author [1], that any Dehn-derived example is in fact of type $M_{H}, M_{\times I}$ or $M_{\text {hybrid }}$. Finally, in Section 6 we verify that all of the old examples that are listed in [5] are of Hempel distance 2, whereas at least some Dehn-derived examples are of distance 3. (It is easy to see that all Dehn-derived examples are of distance no more than 3.)

Acknowledgement The second author was partially supported by an NSF grant.

\section{Dehn derived multiple splittings}

A primitive $k$-tuple of curves in the boundary of a genus $g$ handlebody $H$ is a collection $\lambda_{1}, \ldots, \lambda_{k} \subset \partial H$ of $k \leq g$ disjoint simple closed curves so that, for some 
properly embedded collection $D_{1}, \ldots, D_{k}$ of disks in $H,\left|\lambda_{i} \cap D_{j}\right|=\delta_{i j}, 1 \leq i, j \leq k$. It is easy to see that the closed complement in $H$ of such a collection of meridian disks is a genus $g-k$ handlebody. In particular, if $k=g$ then $\lambda_{1}, \ldots, \lambda_{g}$ is called a complete set of primitive curves and the corresponding collection of disks $D_{1}, \ldots, D_{g}$ is called a complete set of meridian disks. The closed complement of a complete set of meridian disks in $H$ is a 3 -ball.

Suppose $\Lambda=\lambda_{1}, \ldots, \lambda_{k} \subset \partial H$ is a primitive $k$-tuple of curves in $H$ and let $\alpha_{1}, \ldots, \alpha_{k}$ be the properly embedded collection of curves in $H$ obtained by pushing $\Lambda$ slightly into the interior of $H$. We can view $H$ as the boundary connect sum of a genus $g-k$ handlebody $H^{\prime}$ and $k$ solid tori $W_{1}, \ldots, W_{k}$, with $\lambda_{i}$ a longitude of $W_{i}$ and so $\alpha_{i}$ a core curve of $W_{i}$. Then Dehn surgery on $\alpha_{i} \subset W_{i}$ still gives a solid torus. Hence any Dehn surgery on the family of curves $\alpha_{1}, \ldots, \alpha_{k}$ leaves $H$ still a handlebody.

Definition 2.1 Suppose $M_{0}=H_{a} \cup_{S} H_{b}$ is a Heegaard splitting of a closed 3manifold $M_{0}$. A simple closed curve $\lambda \subset S$ is doubly primitive if $\lambda$ is a primitive curve in both handlebodies $H_{a}$ and $H_{b}$.

Suppose $M_{0}$ is a closed orientable 3-manifold and that $M_{0}=H_{a} \cup_{S} H_{b}$ is a genus 2 Heegaard splitting of $M_{0}$. Suppose further that $\lambda_{1}, \lambda_{2} \subset S$ are two disjoint doubly primitive curves in $S$.

Proposition 2.2 Suppose $M$ is a manifold obtained by some specified Dehn surgeries on $\lambda_{1}$ and $\lambda_{2}$. For $i=1,2$, let $A_{i}$ (resp. $B_{i}$ ) be the manifold obtained from the handlebody $H_{a}$ (resp. $H_{b}$ ) by pushing the curve $\lambda_{i} \operatorname{into} \operatorname{int}\left(H_{a}\right)\left(\operatorname{resp} \operatorname{int}\left(H_{b}\right)\right)$ and performing the specified Dehn surgery on the curve.

Then $A_{1} \cup_{S} B_{2}$ and $A_{2} \cup_{S} B_{1}$ are two (possibly different) genus 2 Heegaard splittings of $M$.

Proof $A_{i}$ (resp. $B_{i}$ ) is obtained from $H_{a}$ (resp. $H_{b}$ ) by Dehn surgery on a pushed in copy $\alpha_{i}$ of a single primitive curve in $S$. It was just observed that this makes each $A_{i}\left(\right.$ resp. $\left.B_{i}\right)$ a handlebody.

Definition 2.3 Two genus 2 Heegaard splittings $X \cup_{Q} Y$ and $A \cup_{P} B$ of a closed 3-manifold $M$ are called Dehn derived (from the splitting $M_{0}=H_{a} \cup_{S} H_{b}$ via $\lambda_{1} \cup \lambda_{2} \subset S$ ) if the two splittings are created as in Proposition 2.2.

Corollary 2.4 Suppose $M=A \cup_{P} B=X \cup_{Q} Y$ are a Dehn-derived pair of Heegaard splittings. Then the two hyperelliptic involutions of $M$, one determined by the Heegaard splitting $A \cup_{P} B$ and the other by the Heegaard splitting $X \cup_{Q} Y$, coincide. 
Proof Let $M_{0}=H_{a} \cup_{S} H_{b}$ be the Heegaard split 3-manifold from which the two splittings of $M$ are Dehn derived, via $\lambda_{1} \cup \lambda_{2} \subset S$. The hyperelliptic involution preserves the isotopy class (though perhaps reversing the orientation) of any simple closed curve in $S$. We may then position $\lambda_{i}$ so that the curves are preserved (perhaps reversing orientation) by the hyperelliptic involution on $M_{0}=H_{a} \cup_{S} H_{b}$. Then the hyperelliptic involution on $M_{0}$ naturally induces a single hyperelliptic involution on $M$.

\section{A simple set of examples}

It is not immediately obvious how to create examples of a Dehn-derived pair of splittings or, very naively, whether examples even exist. In this section we present and briefly discuss an important concrete class of examples.

Consider a genus 2 handlebody $H$, constructed from two 0 -handles by connecting them with three 1 -handles. With this structure $H$ has a natural $\mathbb{Z}_{3}$ symmetry, shown as $\frac{2 \pi}{3}$ rotation about the green axis in Figure 1 . Let $\lambda_{1} \subset \partial H$ be the red curve shown in the figure and $\lambda_{2}, \lambda_{3}$ be the other two simple closed curves to which $\lambda_{1}$ is carried by the $\mathbb{Z}_{3}$ symmetry. Then each $\lambda_{i}$ is a primitive curve on $\partial H$ and, indeed, any two of the curves, say $\lambda_{1}, \lambda_{2}$ constitute a complete set of primitive curves (that is, a primitive pair). In this case the corresponding pair of meridian disks are the meridian disks of the two 1-handles through which $\lambda_{3}$ passes.

Let $\bar{H}$ be the genus 3 handlebody obtained by removing from $H$ a neighborhood of the arc in which the axis of symmetry intersects one of the 0 -handles. It is easy to see that in $\bar{H}$ the collection $\lambda_{1}, \lambda_{2}, \lambda_{3} \subset \partial \bar{H}$ is a complete set of primitive curves, that is a primitive 3-tuple.

To construct some Dehn-derived pairs of Heegaard splittings, begin with two genus 2 handlebodies $A$ and $B$, on each of whose boundaries lie three disjoint simple closed curves corresponding to $\lambda_{1}, \lambda_{2}, \lambda_{3} \subset \partial H$. Let $\lambda_{i a} \subset \partial A$ (resp. $\lambda_{i b} \subset \partial B$ ) be the curve corresponding to $\lambda_{i}$ in $A$ (resp. $B$ ), for each $1 \leq i \leq 3$. Adopting (for comparison purposes) notation from [5, Section 4.2], let $\alpha_{n a}, \alpha_{s a}, \rho_{a} \subset A$ be the triple of curves obtained by pushing $\lambda_{1 a}, \lambda_{2 a}, \lambda_{3 a}$ into the interior of $A$ and let $\alpha_{n b}, \alpha_{s b}, \rho_{b} \subset B$ be the triple of curves obtained by pushing $\lambda_{1 b}, \lambda_{2 b}, \lambda_{3 b}$ into the interior of $B$. Let $N$ be a manifold constructed by identifying an annular neighborhood of $\lambda_{1 a}$ in $\partial A$ with an annular neighborhood of $\lambda_{1 b}$ in $\partial B$ and an annular neighborhood of $\lambda_{2 a}$ in $\partial A$ with an annular neighborhood of $\lambda_{2 b}$ in $\partial B$. (After the identification, call the annuli $\mathcal{A}_{n}$ and $\mathcal{A}_{s}$ with core curves $\lambda_{1}, \lambda_{2}$ respectively.) Then identify the two 4-punctured spheres $\partial A-\left(\mathcal{A}_{n} \cup \mathcal{A}_{s}\right)$ and $\partial B-\left(\mathcal{A}_{n} \cup \mathcal{A}_{s}\right)$ by any homeomorphism. 


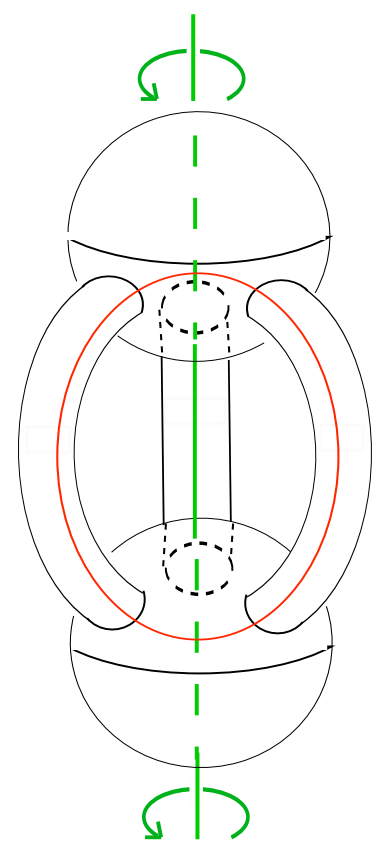

Figure 1

This construction defines a genus 2 Heegaard structure on $N$, of course, but it also defines a genus 2 Heegaard splitting on $M_{0}$, the manifold obtained from $N$ by arbitrary Dehn surgery on just the two curves $\rho_{a} \subset A$ and $\rho_{b} \subset B$, for surgery on these pushedin primitive curves leaves $A$ and $B$ still handlebodies, handlebodies which we denote respectively $H_{a}$ and $H_{b}$. What's more, the curves $\lambda_{1}, \lambda_{2}$ are each primitive in both $H_{a}$ and $H_{b}$ (though they are not necessarily a primitive pair in either). Thus the Heegaard splitting $M_{0}=H_{a} \cup H_{b}$ gives rise to two potentially different genus 2 Heegaard structures on any manifold $M_{H}$ that is obtained by simultaneously doing further Dehn surgery on the two curves $\lambda_{1}, \lambda_{2}$. That is, a manifold $M_{H}$ obtained by arbitrary Dehn surgery on all four curves $\lambda_{1}, \lambda_{2}, \rho_{a}, \rho_{b} \subset N$ has two possibly distinct genus 2 Heegaard splittings, Dehn derived from the Heegaard splitting $M_{0}=H_{a} \cup H_{b}$. One Heegaard structure $M_{H}=A_{1} \cup B_{2}$ is obtained by pushing $\lambda_{1}$ to $\alpha_{n a} \subset \operatorname{int} A$ and $\lambda_{2}$ to $\alpha_{s b} \subset$ int $B$ before doing Dehn surgeries on the four curves; the other $M_{H}=A_{2} \cup B_{1}$ is obtained by pushing $\lambda_{1}$ to $\alpha_{n b} \subset \operatorname{int} B$ and $\lambda_{2}$ to $\alpha_{s a} \subset \operatorname{int} A$ before doing the Dehn surgeries. In each case, exactly two of the four Dehn surgered curves lie in each handlebody $A$ and $B$ before the Dehn surgery, and in that handlebody are a pushed-in primitive pair. 
Proposition 3.1 The two Heegaard splittings $A_{1} \cup B_{2}$ and $A_{2} \cup B_{1}$ of $M_{H}$ become isotopic after at most a single stabilization.

Proof Let $\bar{A}$ and $\bar{B}$ be the genus 3 handlebodies derived from $A$ and $B$ respectively, just as $\bar{H}$ was derived from $H$. Here is a natural genus 3 Heegaard splitting of $M_{H}$ : in contrast to the construction above, push both $\lambda_{1}$ to $\alpha_{n a}$ and $\lambda_{2}$ to $\alpha_{s a}$, so both curves (as well as $\rho_{a}$ ) lie in $A$ before doing the Dehn surgeries. Although $A$ may no longer be a handlebody after the Dehn surgeries, it follows from the discussion above that the result on $\bar{A}$ of the surgery on the three curves $\alpha_{n a}, \alpha_{s a}, \rho_{a} \subset \bar{A} \subset A$ is still a genus three handlebody $\overline{A^{\prime}}$. The complement of $\overline{A^{\prime}}$ in $M_{H}$ is also a handlebody $B^{\prime}$ : a single 1 -handle is added to $B$ and surgery is done on the single curve $\rho_{b} \subset B$. Thus $M_{H}=\bar{A}^{\prime} \cup B^{\prime}$ is a genus 3 Heegaard splitting of $M_{H}$.

It's fairly easy to see that this Heegaard splitting is a stabilization of $A_{1} \cup B_{2}$ (and so, symmetrically, $A_{2} \cup B_{1}$ ). Indeed, an alternate way to construct $\overline{A^{\prime}} \cup B^{\prime}$ is to begin with $A_{1} \cup B_{2}$ and add to $A_{1}$ (and so subtract from $B_{2}$ ) a regular neighborhood of the curve $\alpha_{s b} \subset \operatorname{int}(B)$ and a straight arc from $\partial B$ to $\alpha_{s b}$. From this point of view, the inclusion $B^{\prime} \subset B_{2}$ defines a genus 3 Heegaard splitting of the genus 2 handlebody $B_{2}$, and any such Heegaard splitting is necessarily stabilized (see the paper by the second author and Thompson [8, Lemma 2.7]). The pair of stabilizing disks are also a pair of stabilizing disks for $\overline{A^{\prime}} \cup B^{\prime}$

\section{A second construction, and a hybrid}

Here is another natural, but less naive, way to find disjoint pairs of primitive curves on the boundary of a genus 2 handlebody and so to create a Dehn-derived pair of Heegaard splittings. Let $F$ denote a torus with the interior of a disk removed. Then $F \times I$ is a genus 2 handlebody. For $\gamma$ any properly embedded essential simple closed curve in $F, \gamma \times\{0\}$ (or symmetrically $\gamma \times\{1\}$ ) is a primitive curve in the handlebody $F \times I$. Indeed, for $\delta$ a properly embedded arc in $F$ intersecting $\gamma$ once, $\delta \times I$ is a meridian disk in $F \times I$ that intersects $\gamma \times\{0\}$ exactly once.

Following this observation, and the example of the previous section, here is a recipe for constructing candidate 3-manifolds. Begin with two copies $A$ and $B$ of the surface $F$ and choose two essential (not necessarily disjoint) simple closed curves $\alpha_{0}, \alpha_{1} \subset A$ and two essential (not necessarily disjoint) simple closed curves $\beta_{0}, \beta_{1} \subset B$. Let $\lambda_{0 a}=\alpha_{0} \times\{0\} \subset \partial(A \times I), \lambda_{1 a}=\alpha_{1} \times\{1\} \subset \partial(A \times I), \lambda_{0 b}=\beta_{0} \times\{0\} \subset \partial(B \times$ $I), \lambda_{1 b}=\beta_{1} \times\{1\} \subset \partial(B \times I)$. Identify an annular neighborhood of $\lambda_{0 a}$ in $A \times\{0\}$ with an annular neighborhood of $\lambda_{0 b}$ in $B \times\{0\}$ and call the core curve of the resulting 
annulus $\lambda_{0}$. Similarly identify an annular neighborhood of $\lambda_{1 a}$ in $A \times\{1\}$ with an annular neighborhood of $\lambda_{1 b}$ in $B \times\{1\}$ and call the core curve of the resulting annulus $\lambda_{1}$. Complete the identification of $\partial(A \times I)$ with $\partial(B \times I)$ along the remaining 4punctured sphere arbitrarily. Call the resulting closed 3-manifold $M_{0}$, with Heegaard splitting $M_{0}=(A \times I) \cup(B \times I)$.

The 3-manifold $M_{\times I}$ obtained from $M_{0}$ by doing arbitrary Dehn surgeries to the simple closed curves $\lambda_{0}$ and $\lambda_{1}$ has a Dehn-derived pair of Heegaard splittings: one comes from first pushing $\lambda_{0}$ into $A \times I$ and $\lambda_{1}$ into $B \times I$ before the Dehn surgery, the other comes from first pushing $\lambda_{1}$ into $A \times I$ and $\lambda_{0}$ into $B \times I$ before the Dehn surgery.

Remarks on stabilization It is not apparent to us that a single stabilization will make the two Dehn-derived splittings of $M_{\times I}$ equivalent. The argument of Proposition 3.1 does not immediately carry over: if both curves $\lambda_{0 a}$ and $\lambda_{1 a}$ are pushed into $A \times I$ there is no apparent arc so that the complement $\overline{A \times I}$ of a neighborhood of the arc in $A \times I$ is a genus 3 handlebody after an arbitrary Dehn surgery on the pushed in $\lambda_{0 a}$ and $\lambda_{1 a}$. If there is a proper arc $\gamma$ in $A$ that intersects both curves $\alpha_{0} \subset A$ and $\alpha_{1} \subset A$ in a single point, then the complement $\overline{A \times I}$ after pushing the interior of $\gamma$ into $A \times I$ is a genus 3 handlebody, and so a single stabilization suffices, but having such an arc $\gamma$ is not the general situation. (What is required for such an arc $\gamma$ to exist is that the slopes of $\alpha_{0} \alpha_{1}$ in $A$ are a distance at most two apart in the Farey graph (see Minsky [4, Figure 1]). In that case $\gamma$ has the slope that is incident to the slopes of both $\alpha_{0}$ and $\alpha_{1}$ in the Farey graph.)

On the other hand, it is relatively easy to show that two stabilizations suffice to make the two splittings equivalent. To see this, push both $\lambda_{0}$ and $\lambda_{1}$ into $A \times I$ and connect them to respectively $A \times\{0\}$ and $A \times\{1\}$ by straight arcs. Then add a regular neighborhood of the arcs and of the pushed in curves $\lambda_{0}$ and $\lambda_{1}$ to $B \times I$ to create a genus 4 handlebody $\overline{B \times I}$ and simultaneously subtract the regular neighborhood from $A \times I$ to get the genus 4 handlebody $\overline{A \times I}$. The resulting genus 4 Heegaard splitting $M_{0}=\overline{A \times I} \cup \overline{B \times I}$ becomes a Heegaard splitting $H_{a}^{+} \cup H_{b}^{+}$of $M_{\times I}$ after the prescribed Dehn surgery on $\lambda_{0}$ and $\lambda_{1}$. Using the argument of Proposition 3.1 it is easy to see that the Heegaard splitting $H_{a}^{+} \cup H_{b}^{+}$destablizes to the genus 3 splitting obtained by instead pushing $\lambda_{0}$ into $B \times I$ and then adding to $B \times I$ a regular neighborhood of $\lambda_{1} \subset(A \times I)$ and a straight arc attaching it to $A \times\{1\}$. The argument of Proposition 3.1 applied again shows that this Heegaard splitting destabilizes to the genus 2 splitting in which $\lambda_{0}$ is pushed into $B \times I$ and $\lambda_{1}$ into $A \times I$, one of the Dehn-derived splittings. But this destabilization process is clearly symmetric: we could equally well have destabilized to the other genus 2 splitting, in which $\lambda_{0}$ is pushed into $A \times I$ and $\lambda_{1}$ into $B \times I$, and this is the other Dehn-derived splitting. 
A further, call it a hybrid example of a Dehn-derived pair of splittings comes by combining the two constructions above: Identify annular neighborhoods of $\lambda_{1}, \lambda_{2} \subset \partial H$ from Section 3 with annular neighborhoods of $\lambda_{0 b}, \lambda_{1 b} \subset \partial(B \times I)$ and identify the rest of $\partial H$ with the rest of $\partial(B \times I)$ in any way. This gives a closed 3-manifold $N$ with a Heegaard splitting $H \cup(B \times I)$. Let $M_{0}$ be a 3-manifold obtained by doing an arbitrary Dehn surgery on $\lambda_{3} \subset \partial H$, after pushing it into int $(H)$. Then $M_{0}$ has the genus 2 Heegaard splitting (exploiting the notation used above) $M_{0}=H_{a} \cup(B \times I)$. Let $M_{\text {hybrid }}$ be a closed 3-manifold obtained from $M_{0}$ by arbitrary Dehn surgeries on the two remaining curves $\lambda_{1}, \lambda_{2} \subset \partial H_{a} \subset M_{0}$. The Dehn-derived pair of Heegaard splittings for $M_{\text {hybrid }}$ is obtained by alternatively pushing $\lambda_{1}$ into $H_{a}$ and $\lambda_{2}$ into $B \times I$ or vice versa. A single stabilization suffices to make the two splittings equivalent, essentially by the same argument as for $M_{H}$, in Proposition 3.1.

\section{A taxonomy of Dehn-derived splittings}

Sections 3 and 4 give concrete examples of pairs of Dehn-derived fillings. In this section we show that these examples in fact constitute all pairs of Dehn-derived splittings. The argument exploits Berge's classification of pairs of primitive curves on genus 2 handlebodies [1], though the classification here is slightly different.

Let $H$ be a genus 2 handlebody, with $\lambda_{1}, \lambda_{2}, \lambda_{3} \subset \partial H$ the disjoint simple closed curves described in Section 3. Denote by $\rho$ the curve in the interior of $H$ obtained by pushing $\lambda_{3}$ into $H$ and let $H_{\text {surg }}$ denote the handlebody obtained from $H$ by a specified Dehn surgery on $\rho \subset \operatorname{int}(H)$. As in Section 4, let $F$ denote a torus with the interior of a disk removed.

Proposition 5.1 (Berge) Suppose $\alpha$ and $\beta$ are disjoint non-parallel primitive curves on the boundary of a genus 2 handlebody $H$. Then either

(A) there is a Dehn surgery on $\rho \subset H$ and a homeomorphism $h: H \rightarrow H_{\text {surg }}$ so that $h(\alpha)=\lambda_{1} \subset \partial H_{\text {surg }}$ and $h(\beta)=\lambda_{2} \subset \partial H_{\text {surg }}$ or

(B) there is a homeomorphism $h: H \rightarrow F \times I$ so that $h(\alpha) \subset F \times\{0\}$ and $h(\beta) \subset$ $F \times\{1\}$.

Proof This classification is a variant of that described in [1]. The Type II pair there, as well as some pairs of Type I, are exactly as described in alternative (B). The interest is in the third example of a Type I pair, in [1, Lemma 3.8 (3) via Figure 3]. In that example, $H$ is viewed as divided into two solid tori by a separating disk $D$; let $\lambda_{a}$ and $\lambda_{b}$ be longitudes of the two solid tori into which $D$ divides $H$. Then $\beta$ is parallel to 
$\lambda_{b}$, and $\alpha$ is the band sum, via a band that crosses $D$ once, of $\lambda_{b}$ with a torus knot on the solid torus containing $\lambda_{a}$. This picture is equivalent to letting $\alpha$ be the band sum $\lambda_{a} \# \lambda_{b}$ (through $D$ ) of $\lambda_{b}$ with $\lambda_{a}$, and then performing a Dehn surgery on a disjoint copy of $\lambda_{a}$ that has been pushed into $H$, to become a core of the solid torus on which $\lambda_{a}$ lies. Now translate: relabel $\lambda_{b} \subset \partial H$ as $\lambda_{2}$ and $\lambda_{a} \subset \partial H$ as $\lambda_{3}$. Then $\lambda_{a} \# \lambda_{b}$ corresponds to $\lambda_{1}$. The construction just described is then to push $\lambda_{3}$ into the interior of $H$ and perform some surgery to get $H_{\text {surg }}$. Afterwards $\alpha$ corresponds to $\lambda_{1} \subset \partial H_{\text {surg }}$ and $\beta$ corresponds to $\lambda_{2} \subset \partial H_{\text {surg }}$. This is exactly alternative (A).

Following Proposition 5.1 there is a fairly clear description of the cases of multiple Heegaard splittings that are missing from [5]. According to [7] the only missing cases are pairs of splittings that are Dehn-derived from an initial splitting $H_{A X} \cup H_{B Y}$ of a manifold $M_{0}$. First determine which of alternatives (A) and (B) apply to the pairs of surgery curves as they lie on the boundaries of the respective handlebodies: $\left\{a_{1}, c_{2}\right\} \subset H_{A X}$ or $\left\{a_{2}, c_{1}\right\} \subset H_{B Y}$. If both are of type (A) then the pair of splittings is Dehn-derived as in the construction of $M_{H}$ in Section 3. If both are of type (B) then the pair of splittings is Dehn-derived as in the construction of $M_{\times I}$ in Section 4. If one is of type (A) and one of type (B) then the pair of splittings is Dehn-derived as in the construction of $M_{\text {hybrid }}$ in Section 4.

It is worth mentioning that there is another view of a pair of primitive curves lying on a handlebody as in (A) of Proposition 5.1, a view that more closely resembles that in (B): Let $\alpha, \beta, \gamma$ be simple closed curves in $F$ so that each pair of curves intersects in exactly one point. (For example, choose curves in $F$ of slopes $0,1, \infty$.) Then it is fairly easy to see that the three curves $\alpha \times\{0\}, \beta \times\{1\}, \gamma \times\left\{\frac{1}{2}\right\}$ lie in the handlebody $F \times I$ just as $\lambda_{1}, \lambda_{2}, \rho$ lie in $H$ in the description preceding Proposition 5.1. So the primitive curves in description (A) can be made to look like a special case of those in description (B), but with the cost that an extra Dehn surgery has to be performed on a specific curve in the interior of $F \times I$. This is the twisted product view of [1,3.2].

\section{Distance}

It would seem possible that the Dehn-derived pairs of Heegaard splittings exhibited above could coincidentally all be contained among the examples already listed in [5], for there is no claim that the types of examples of multiple Heegaard splittings we have offered here and in [5] do not overlap. But in fact there is an invariant which does show that at least some Dehn-derived pairs of Heegaard splittings described above did not already occur in a different guise in [5]. This invariant had not yet been introduced when [5] was written and is called the (Hempel) distance of the Heegaard splitting (see Hempel [3]). We briefly review: 
Definition 6.1 A Heegaard splitting $H_{1} \cup_{S} H_{2}$ has Hempel distance at most $n$ if there is a sequence $c_{0}, \ldots, c_{n}$ of essential simple closed curves in the splitting surface $S$ so that

- $\quad$ for each $i=1, \ldots, n, c_{i} \cap c_{i-1}=\varnothing$

- $c_{0}$ bounds a disk in $H_{1}$

- $c_{n}$ bounds a disk in $\mathrm{H}_{2}$

If the splitting has distance $\leq n$ but not $\leq n-1$, then the distance $d(S)=n$.

A Heegaard splitting of distance 0 is called reducible; one of distance $\leq 1$ is called weakly reducible. Any Heegaard splitting of a reducible manifold is reducible. A Heegaard splitting of distance $\leq 2$ is said to have the disjoint curve property (see Thompson [9]); any Heegaard splitting of a toroidal 3-manifold has the disjoint curve property [3;9]. A weakly reducible genus 2 Heegaard splitting is also reducible, so an irreducible Heegaard splitting of genus 2 has distance at least 2 [9].

In the other direction we have:

Proposition 6.2 Suppose the manifold $M$ has a Dehn-derived pair of Heegaard splittings. Then each of these Heegaard splittings has Hempel distance at most 3 .

Proof Suppose the splittings are Dehn-derived from a splitting $M_{0}=H_{a} \cup_{S} H_{b}$ via the disjoint pair of simple closed curves $\lambda_{1}, \lambda_{2} \subset S$. With no loss of generality, consider the splitting $M=A \cup_{S} B$ obtained by pushing $\lambda_{1}$ into $\operatorname{int}\left(H_{a}\right)$ and $\lambda_{2}$ into $\operatorname{int}\left(H_{b}\right)$ before doing Dehn surgery on the $\lambda_{i}$. Since $\lambda_{1}$ is primitive in $H_{a}$ there is a properly embedded essential disk $D_{a} \subset H_{a}$ that is disjoint from $\lambda_{1}$. (For example $D_{a}$ can be obtained from a meridian disk $D_{1} \subset H_{a}$ that intersects $\lambda_{1}$ in a single point by band-summing together two copies of $D_{1}$ along a subarc of $\lambda_{1}-D_{1}$.) $D_{a}$ is then also disjoint from the curve $\alpha_{1} \subset H_{a}$ obtained by pushing $\lambda_{1} \operatorname{into} \operatorname{int}\left(H_{a}\right)$, so $D_{a}$ remains intact as a meridian of $A$ after surgery on $\alpha_{1}$. Hence $\partial D_{a}$ and $\lambda_{1}$ are disjoint curves in $\partial A$.

Symmetrically, there is a meridian $D_{b} \subset B$ so that $\partial D_{b}$ and $\lambda_{2}$ are disjoint curves in $\partial B$. Then the sequence $\partial D_{a}, \lambda_{1}, \lambda_{2}, \partial D_{b}$ shows that the splitting $A \cup_{S} B$ has distance at most 3 .

Proposition 6.3 All examples of multiple Heegaard splittings appearing in [5, Section 4] have Hempel distance $\leq 2$. 
Proof Following the comments above we can restrict attention to irreducible, atoroidal manifolds. We briefly run through the examples as they appear in [5, Section 4]. Typically the description of an example $\mathrm{H}_{1} \cup_{S} \mathrm{H}_{2}$ in [5] consists of two parts: A collection of annuli $\mathcal{A} \subset S$ along which $\partial H_{1}$ and $\partial H_{2}$ are identified, followed by an arbitrary identification of $\partial H_{1}-\mathcal{A}$ with $\partial H_{2}-\mathcal{A}$. From this point of view the simple closed curves $\partial \mathcal{A} \subset S$ that separate one sort of region from the other will be called the seams of the Heegaard splitting. We will observe that in [5] some seam is always disjoint from an essential disk in $H_{1}$ and an essential disk in $H_{2}$. This demonstrates that the splitting has the disjoint curve property and so has distance $\leq 2$.

To be specific: In [5, Subsection 4.1], [5, Subsection 4.2, Variation 1] and [5, Subsection 4.4, Variations 1 and 2], the meridians of the 1-handles $e_{a}$ and $e_{b}$ are disjoint from the seams. [5, Subsection 4.2, Variation 2] is slightly more complicated. It is a bit like the construction in Section 3 above: Handlebodies $A$ and $B$ are identified along neighborhoods of all three curves $\lambda_{i}, i=1,2,3$, Dehn surgery is done to all three, with $\lambda_{1}, \lambda_{2}$ pushed into $A$ and $\lambda_{3}$ into $B$ (then vice versa). But there is a meridian of $A$ disjoint from $\lambda_{1}$ and $\lambda_{2}$ and a meridian of $B$ disjoint from $\lambda_{1}$ and $\lambda_{3}$, so a seam parallel to $\lambda_{1}$ demonstrates that the splitting of [5, Subsection 4.2, Variation 2] has the disjoint curve property.

The manifolds in [5, Subsection 4.3] and [5, Subsection 4.4, Variations 3, 4, and 7] are all toroidal, so they are of distance $\leq 2$. What remains are [5, Subsection 4.4, Variations 5 and 6] and we adopt the terminology there. In Variation 5, with, say, $\rho_{a} \subset A_{-}$, the seams that are the boundary of the 4-punctured sphere $\partial A_{-} \cap \partial \Gamma$ are all disjoint from the meridian of the 1-handle $e_{b} \subset B$ and, in $A_{-}$, any one of these seams together with $\rho_{a}$ lie in $A_{-}$as two of the $\lambda_{i}$ 's of Section 3 above lie in $H$. In particular, there is a meridian of $A_{-}$disjoint from both the seam and from $\rho_{a}$. Thus that seam again illustrates that the splitting has the disjoint curve property.

The argument for Variation 6 is much the same. First note that if, in that Variation, Dehn surgeries are done on two curves parallel to $\sigma$, then the resulting manifold has a Seifert piece and so has distance $\leq 2$. So the only change we need to consider from Variation 5 is Dehn surgery on a single curve parallel to $\sigma$. If that curve lies in $B$ the argument for Variation 5 suffices; if it is in $A_{-}$this merely forces us to pick a specific seam in the argument for Variation 5, a seam parallel to the new surgery curve.

In contrast, some of the examples constructed in this paper can be shown to have distance 3, so they cannot have appeared in any case considered in [5]. See [2] (also [6]) for details. 


\section{References}

[1] J Berge, A classification of pairs of disjoint nonparallel primitives in the boundary of a genus two handlebody arXiv:0910.3038

[2] J Berge, A closed orientable 3-manifold with distinct distance three genus two Heegaard splittings, to appear

[3] J Hempel, 3-manifolds as viewed from the curve complex, Topology 40 (2001) 631-657 MR1838999

[4] Y N Minsky, The classification of punctured-torus groups, Ann. of Math. (2) 149 (1999) 559-626 MR1689341

[5] H Rubinstein, M Scharlemann, Genus two Heegaard splittings of orientable threemanifolds, from: "Proceedings of the Kirbyfest (Berkeley, CA, 1998)", Geom. Topol. Monogr. 2, Geom. Topol. Publ., Coventry (1999) 489-553 MR1734422

[6] M Scharlemann, Berge's distance 3 pairs of genus 2 Heegaard splittings arXiv: 1002.4887

[7] M Scharlemann, Genus two Heegaard splittings: an omission, from: "Proceedings of the Kirbyfest (Berkeley, CA, 1998)", Geom. Topol. Monogr. 2, Geom. Topol. Publ., Coventry (1999) 577-581

[8] M Scharlemann, A Thompson, Heegaard splittings of (surface) $\times I$ are standard, Math. Ann. 295 (1993) 549-564 MR1204837

[9] A Thompson, The disjoint curve property and genus 2 manifolds, Topology Appl. 97 (1999) 273-279 MR1711418

Independent, Madison 53711 Wisconsin, USA

Department of Mathematics, University of California

Santa Barbara CA 93106, USA

jberge@charter.net, mgscharl@math.ucsb.edu

Received: 10 May $2010 \quad$ Revised: 11 April 2011 